\title{
Preliminary Evidence of Increased Pain and Elevated Cytokines in Fibromyalgia Patients with Defective Growth Hormone Response to Exercise
}

\author{
Rebecca L. Ross ${ }^{*}, 1$, Kim D. Jones ${ }^{1,3}$, Robert M. Bennett ${ }^{1,3}$, Rachel L. Ward ${ }^{1}$, Brian J. Druker ${ }^{2}$ and \\ Lisa J. Wood ${ }^{1,2}$ \\ ${ }^{1}$ Oregon Health \& Science University, School of Nursing, 3455 SW US Veterans Hospital Road, Portland, OR 97239, \\ USA \\ ${ }^{2}$ Oregon Health \& Science University Cancer Institute, Division of Hematology and Medical Oncology, 3181 SW Sam \\ Jackson Park Rd., Portland, OR 97239, USA \\ ${ }^{3}$ Oregon Health \& Science University, School of Medicine, 3181 SW Sam Jackson Park Road, Portland, OR 97239, USA
}

\begin{abstract}
Mounting evidence suggests fibromyalgia (FM) symptoms are influenced by dysfunction of the hypothalamicpituitary-hormonal axes (HPHA) and the immune response system. The predominant FM symptoms of widespread pain, fatigue, sleep disturbance, depression, stiffness and exercise intolerance are related to abnormal levels of growth hormone $(\mathrm{GH})$ and are reminiscent of "sickness behavior"; a syndrome initiated by the production of pro-inflammatory cytokines in response to various stressors. Cognizant of the reciprocal relationship between HPHA activity and the immune response system, we hypothesized that serum cytokine levels and FM symptom severity would be higher in FM patients with defective growth hormone response to exhaustive exercise compared to those without. Outpatients with FM ( $\mathrm{n}=165$ ) underwent a Modified Balke Treadmill Protocol and GH response to exhaustive exercise was measured in peripheral blood samples. Levels of IL-1 $\alpha$, IL-1 $\beta$, IL-1RA, IL-6, IL-8, IL-10, and TNF- $\alpha$ were measured from stored serum on a subset of 24 participants (12 with and 12 without normal GH response to exhaustive exercise). FM symptom severity was assessed using the Fibromyalgia Impact Questionnaire (FIQ), number of tender points and cumulative myalgic scores. GH dysfunction was associated with increased pain scores on the FIQ $(p=0.024)$, a greater number of tender points $(p=$ $0.014)$, higher myalgic scores $(\mathrm{p}=0.001)$ and higher pre-exercise levels of inflammatory cytokines IL-1 $\alpha(\mathrm{p}=0.021)$, IL$6(\mathrm{p}=0.012)$, and IL-8 $(\mathrm{p}=0.004)$. These results suggest that a defective growth hormone response to exercise may be associated with increased levels of blood cytokines and pain severity in FM patients.
\end{abstract}

Keywords: Fibromyalgia, cytokines, hypothalamic-pituitary-growth hormone axis, hypothalamic-pituitary-adrenal axis, immune response system, cortisol.

Fibromyalgia (FM) is currently viewed as a common pain syndrome related to dysfunction of central nociceptive sensory processing $[1,2]$. A similar neurosensory dysfunction probably accounts for the common association of FM with many other painful syndromes [3]. However, several of the other symptoms experienced by FM patients, such as mood disorders, fatigue, specific sleep disturbances, stiffness and post-exertional pain cannot be fully explained by the abnormalities of central pain processing. This has led researchers to consider dysfunction of the stress axis, both the autonomic and humeral components, as possibly being relevant to some FM symptoms [4, 5]. While, overall, there are currently no robust biomarkers for FM except for experimental pain testing [6], there is emerging evidence that some patients with FM [7] or widespread pain [8] have a reduced resiliency of the hypothalamic-pituitary-hormonal axes (HPHA) with regard to both the secretion of cortisol and growth hormone $(\mathrm{GH})$ [9]. Previous work from our

*Address correspondence to this author at the Oregon Health \& Science University School of Nursing, Mail Code: SN-ORD, 3455 SW US Veterans Hospital Road, Portland, OR 97239, USA; Tel: 503.494.4326; Fax: 503.418.0903; E-mail: rossre@ohsu.edu group has reported hypothalamic-pituitary-growth-hormone (HPGH) axis with low plasma IGF-1 levels - a measure of 24-hour GH secretion - in about one third of persons with FM [10]. Further experiments have shown that the reduced $\mathrm{GH}$ secretion in FM patients is a result of increased hypothalamic somatostatin tone, which inhibits GH secretion [11]. In addition, depression is associated with increased secretion of corticotropin-releasing hormone (CRH), which in turn stimulates the secretion of somatostatin and cortisol. The HPHA and the immune response system are comprised of multiple homeostatic systems with negative and positive feedback mechanisms for $\mathrm{CRH}$, cortisol, somatostatin, $\mathrm{GH}$, and cytokine release. The balance can be affected uniquely and synergistically by each of the stress, growth and reproductive hormonal axes and cytokines. A visual schematic of the acute and chronic stress response and the relationships between these key hormones is depicted in Fig. (1).

It has been noted that some FM symptoms are reminiscent of "sickness behavior", a syndrome of pain, fatigue, reduced activity, impaired cognition and depressed mood caused by activation of the immune response system 


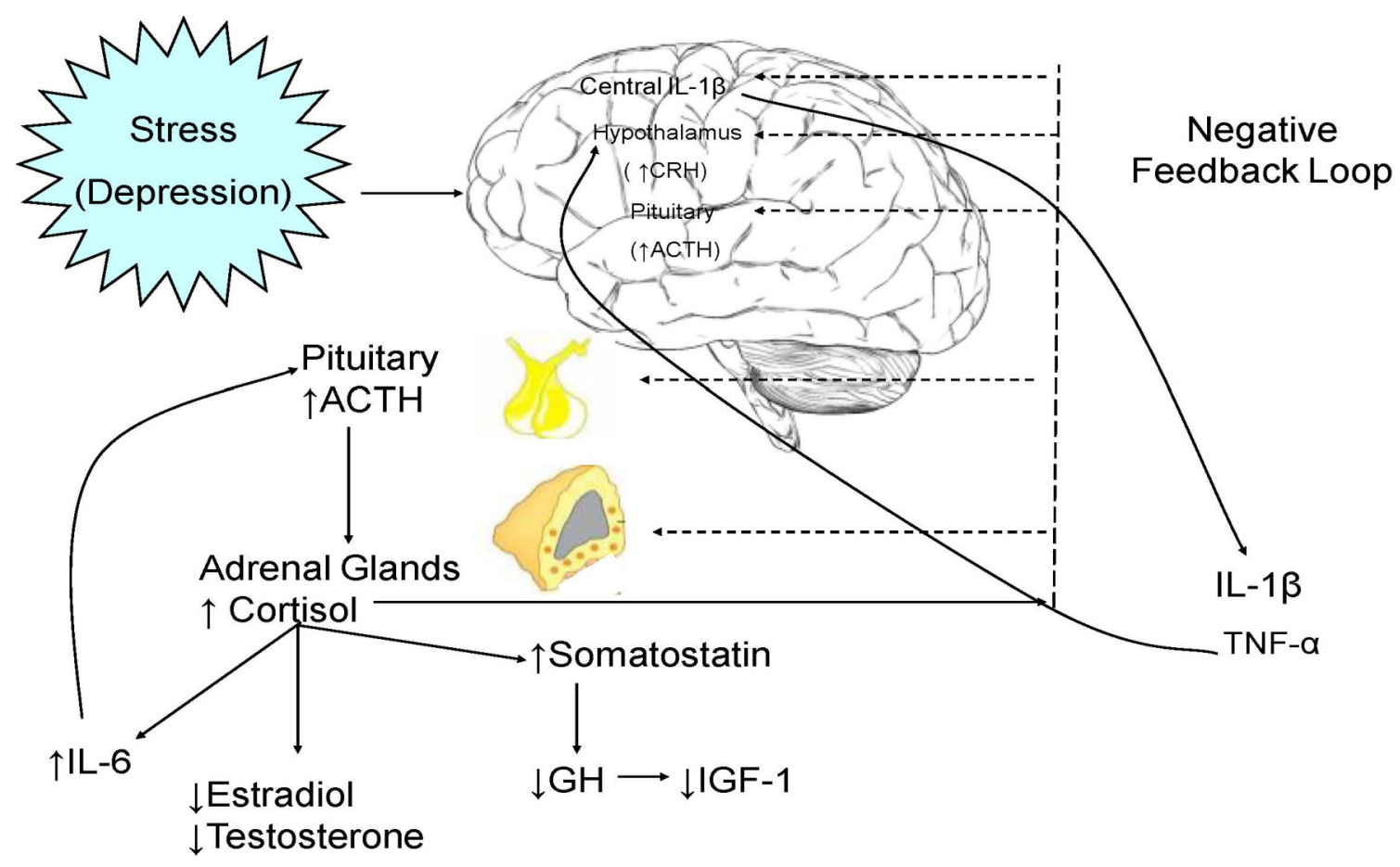

Fig. (1). Effects of chronic stress on biochemical markers of the HPA and HPGH axes and immune response system. Acute and chronic stressors stimulate the release of corticotropin-releasing hormone $(\mathrm{CRH})$ from the hypothalamus which in turn stimulates the pituitary to release adrenocorticotropic hormone (ACTH). ACTH travels thru the circulatory system to the adrenal glands and stimulates the release of cortisol. Stressors also stimulate the release of IL-1 $\beta$ in the brain which initiates the release of TNF- $\alpha$. IL-1 $\beta$ directly activates the HPA axis, culminating in the release of cortisol, which stimulates the production of IL-6. Cortisol also affects the growth and reproductive axes (solid lines indicate activation while dashed lines indicate inhibition).

and characterized by increased production of inflammatory cytokines and chemokines [12,13]. Sickness behavior is usually described as the integrated set of behavioral changes that develop as a result of infection [14]. However, it is now apparent that activation of the immune response system is not limited to infection, but can also occur in chronic stress, obesity, depression, exhaustive exercise and in overtraining syndrome [15-19]. Moreover, there is good evidence to implicate the interaction of inflammatory cytokines with glial cells as a trigger for the development of sickness behavior [20].

Elevated levels of pro-inflammatory cytokines have been reported in diverse circumstances including infections, obesity (especially visceral obesity) [21], excessive exercise (e.g. marathoners) [22, 23], PTSD [24], type-2 diabetes [25], and depression [26]. Although fibromyalgia is generally considered to be a non-inflammatory disorder, recent research has demonstrated the role of spinal neuroimmune responses as contributing to persistent pain [14]. Cytokines and growth factors have been strongly implicated in the generation of pathological pain states throughout the nervous system; in particular, the pro-inflammatory cytokines interlukin-1 beta (IL-1 $\beta$ ) and tumor necrosis factor-alpha $(\mathrm{TNF}-\alpha)$ are up regulated both locally and in the spinal cord in persistent pain [27-30]. In addition, serum levels of IL-8 have been reported to be increased in five FM studies [31$35]$; although two studies failed to confirm these reports [36, 37].

Despite the importance of IL- $1 \beta$, IL- 8 and TNF- $\alpha$ in the development and maintenance of inflammatory pain and in central sensitization, the role that these cytokines play in FM symptomatology has been largely unexplored. Cognizant of the observation that some FM patients have an impaired GH response to the stress of exhaustive exercise [38, 39] and recognizing the reciprocal relationship between the immune response system and HPHA activity [40-42], we hypothesized that serum levels of inflammatory cytokines and chemokines would be elevated in FM patients with a defective GH response to exhaustive exercise. Additionally, since activation of the immune response system triggers sickness behavior, we proposed that FM symptom severity would be higher in FM patients with HPHA dysfunction.

\section{MATERIALS AND METHODOLOGY}

\section{Protocol}

Approval of the protocol, as described elsewhere [38], was obtained from the university's Investigational Review Board and all participants gave written informed consent before any procedures were performed. Participants were selected from a parent study of 165 FM participants who previously underwent the stress of exhaustive exercise using the modified Balke Treadmill protocol. Inclusion and exclusion criteria as previously described [38] included participants who were 18-60 years of age and diagnosed with FM as per the 1990 American College of Rheumatology (ACR) criteria [43].

\section{Modified Balke Treadmill Exercise Protocol [44]}

This protocol was used to induce an acute state of stress. Participants who had fasted overnight were exercised on a 
treadmill with a fixed gradient to the point of volitional exhaustion $\left(\mathrm{VO}_{2} \mathrm{Max}\right)$, which was defined when either of 2 goals was reached: 1) respiratory rate index greater than 1.1 for at least 30 seconds, indicative of anaerobic metabolism, or 2) exhaustion (Borg) scale value of 10 (maximum perceived effort). Electrocardiography and blood pressure monitoring were performed every 5 minutes. All testing was performed between 10:00 AM and 2:00 PM. Since the magnitude of the GH response to exercise is related to the intensity and the duration of exercise, the amount of work performed by each participant during the test was calculated using the following equation: workload $=($ distance/time $) \mathrm{x}$ treadmill grade $\mathrm{x}$ weight.

\section{Blood Collection \& Storage}

Peripheral blood was collected through an indwelling intravenous catheter that was inserted at least $60 \mathrm{~min}$ prior to the pre-exercise blood draw. Samples were drawn immediately before exercise (pre-exercise), immediately after exercise (post-exercise), and 1 hour after the end of exercise (1-hr. post-exercise). The blood was allowed to clot and the resulting serum was stored at $-70 \mathrm{C}$ prior to running batched analyses.

\section{Insulin-like Glucose Factor-1, Growth Hormone \& Cortisol}

Twenty-four hour GH function was measured by proxy via insulin-like glucose factor-1 (IGF-1), a long-term marker of pulsatile GH secretion. Pre-exercise serum samples were analyzed by immunoradiometric assay (IRMA, Diagnostic Systems Laboratories, Webster, TX). Sensitivity of the assay is $0.80 \mathrm{ng} / \mathrm{dL}$. The mean intra-assay coefficients of variation (CV) were $2.6 \%$ and mean inter-assay CV was $4.5 \%$. Data on IGF-1 was categorized as $0=$ low for age and $1=$ expected for age. Growth hormone was measured by a non-competitive chemiluminescence assay (Diagnostic Products, Los Angeles, CA) from all three time points. Sensitivity of this assay is 0.01 $\mathrm{ng} / \mathrm{mL}$; the mean intra-assay $\mathrm{CV}$ is $6.0 \%$ and the mean interassay $\mathrm{CV}$ is $5.8 \%$. Growth hormone response was calculated using the following equation: $\mathrm{GH}^{\text {Post-exercise }}-\mathrm{GH}^{\text {Pre-exercise }}$. Normal GH response to exercise to $\mathrm{VO}_{2} \mathrm{Max}$ is $\geq 5 \mathrm{ng} / \mathrm{mL}$. Cortisol was measured in duplicate by immunoassay (R\&D Systems) according to the manufacturer's protocol. Sensitivity of this assay is $0.01 \mathrm{pg} / \mathrm{mL}$. The inter-assay CV was below $10.5 \%$ and the intra-assay $\mathrm{CV}$ was below $13.4 \%$. The normal resting level range of cortisol is $300-2500 \mathrm{pg} / \mathrm{mL}$.

\section{Inflammatory Markers}

Pre-exercise serum levels of IL-1 $\alpha$, IL-1 $\beta$, IL1RA, IL-6, IL-8, IL-10, and TNF- $\alpha$ were measured using a bead-based immunoflourescence assay (Luminex Inc., Austin, TX). Multiple cytokine analysis kits (LINCOplex kits) were obtained from Linco Research Inc. (St. Charles, MO). Millipore multiscreen 96 well filter plates (Bedford, MA) were used for all multiplex cytokine kits. Assays were run in duplicate according to the manufacturers' protocol. Data were collected and analyzed using the Luminex-100 system Version IS (Luminex, Austin, TX). A four-parameter regression formula was used to calculate the sample concentrations from the standard curves.

\section{Instruments and Other Testing}

Participants completed investigator-conducted evaluations and self-report instruments chosen specifically for their psychometric properties and appropriateness for use in an FM population.

\section{Demographic Data Form}

The Demographic Data Form, an investigator-designed questionnaire, was used to obtain demographic information including age, gender, disability status, marital status, estrogen status, and the year FM was diagnosed by a health care professional.

\section{Revised Beck Depression Inventory (BDI-R)}

The BDI is a well-known, 21-item scale that measures mood and behaviors characteristic of depression [45]. The BDI has been adapted for use with FM patients by removing from the total score 3 items that are characteristic of all FM patients (fatigue, sleep difficulties, and effort required to get things done), and which, therefore, do not correlate well with major depressive disorder. This revision, the BDI-R, was selected because it has better accuracy, sensitivity, and specificity in an FM population than the original [46].

\section{Fibromyalgia Impact Questionnaire (FIQ)}

Fibromyalgia symptoms were measured with the FIQ; a 10 -item instrument that uses a four-point scale to measure physical functioning and a $0-100 \mathrm{~mm}$ Visual Analog Scale (VAS) to measure symptoms of pain $(0=$ no pain and $10=$ very severe pain $)$, fatigue $(0=$ no tiredness and $10=$ extreme tiredness), sleep $(0=$ awakening un-refreshed and $10=$ awakening well-rested), stiffness $(0=$ no stiffness and $10=$ very stiff), anxiety $(0=$ no anxiety and $10=$ very anxious $)$, and depression $(0=$ no depression and $10=$ very depressed $)$, along with levels of disability and overall well-being during the previous week. Total FIQ scores range from 0-100, with higher values indicating a more negative impact of FM [47].

\section{Pain Assessments}

A combination of three pain measures was used: the FIQ pain VAS subscale, the number of tender points, and the Cumulative Myalgic Score (CMS). The $100 \mathrm{~mm}$ VAS for pain rated the participant's perception of pain intensity over the previous 7 days, with anchors at 0 (no pain) and 10 (very severe pain). The CMS is an 18-item scale that diagnoses FM as per the 1990 American College of Rheumatology (ACR) criteria plus rates the amount of pain associated with 4-kilograms of pressure applied to 18 tender points commonly found in FM [43]. Pain severity was measured by having the participants rate their pain level on a $0-3$ scale $(0$ = no pain, $1=$ some pain, 2 = ouch, $3=$ moves away) when 4 $\mathrm{kg}$ of pressure was applied at each site. Higher scores indicate more pain, with a total possible score range of 0 to 54. The number of non-zero scores out of 18 determined the total tender point count. A single examiner performed all tender point evaluations.

\section{Flannigan's Quality of Life Scale (QOLS)}

The QOLS, used to measure broad dimensions of overall quality of life, is a 16-item Likert-type scale that assesses 
multiple areas of well-being and life satisfaction [48]. The concept of QOL is measured on a continuum where $1=$ terrible and $7=$ delighted. The possible range of scores is 16 to 112 , with higher scores indicating better well-being and quality of life. It has been validated in an FM sample with an internal consistency reliability alpha equaling .82 to .88 and test-retest reliability of $.84[49,50]$.

\section{Body Mass Index (BMI)}

Body weight and height were measured in kilograms and meters using a calibrated standing model scale (Detecto). Body mass index (BMI) was calculated using a standard formula of weight in kilograms divided by height in meters squared. Body composition (lean body mass and fat body mass) and percentage of body fat were derived from 7-point skin-fold caliperimetry (chest, axilla, triceps, sub scapula, abdomen, suprailiac, thigh) with a 2 prong spring-loaded Harpenden caliper per standardized anthropomorphic guidelines [51].

\section{Statistical Analysis}

All data analyses were conducted using the Statistical Programs for Social Sciences (SPSS), version 15.0. All analyses of significance $(\mathrm{p} \leq 0.05)$ used a 2 -tail distribution. Group comparisons were preformed for nominal data using the chi-square test or Fisher's exact test for small expected numbers. Student's t-tests were used to compare growth hormone response to exhaustive exercise, FM symptom severity, and biochemical markers between groups. When the assumption of equal variances was violated, a Welch's test was used. Analysis of covariance (ANCOVA) was conducted to measure the effect of workload [workload = (distance/time) $\mathrm{x}$ treadmill grade $\mathrm{x}$ weight], BMI, and percentage of body fat on $\mathrm{GH}$ response to exhaustive exercise between groups with the independent variable having 2 levels (GH responders versus non-responders) and the dependent variable being $\mathrm{GH}$ response $\left(\mathrm{GH}^{\text {Post-exercise }}\right.$ $\left.\mathrm{GH}^{\text {Pre-exercise }}\right)$. Similarly, ANCOVA was used to control for BMI and percentage of body fat in the between group analyses of immune response system markers. Pearson's correlation coefficients were computed among biochemical markers and FM symptoms specific to our a priori hypotheses that were found to differ significantly between groups. While correlational analyses were intended to be exploratory, we computed adjusted Bonferroni $p$ values $(.05 / 14=0.004)$ to allow the reader to further interpret the statistical rigor of the correlations.

\section{RESULTS}

\section{Demographic Characteristics}

The sample consisted of 24 non-Hispanic Caucasian females between the ages of 28 and 60. Interestingly, only 12 of the 165 participants from the parent study mounted a normal GH response to exhaustive exercise $(\geq 5 \mathrm{ng} / \mathrm{dL}$ increase during exercise), and thus restricted the sample size to 12 participants per group. For the comparison group, 12 female participants who did not mount a GH response to exhaustive exercise $(0 \mathrm{ng} / \mathrm{dL}$ increase during exercise) were randomly selected from the remaining 153 participants. No significant differences on demographic features were found between the 141 participants from the parent group and the 24 participants selected for the secondary analysis group $(\mathrm{p}=$
.214 to 1.0$)$. Sera from only seven of the 12 participants with HPHA dysfunction were in sufficient quantity and quality to perform immune response system biomarker analysis. The five participants without sufficient sample to analyze did not significantly differ on demographic characteristics from the seven responders whose sera were analyzed $(p=0.140$ to 1.0). Menopause was more prominent in those with HPHA dysfunction. While groups did not statistically differ on receiving disability wages, only $33.3 \%$ of participants with HPHA dysfunction were able to work outside of the home compared to $85.7 \%$ of those with normal HPHA function ( $\mathrm{p}$ $=0.029$ ). No significant differences existed between the two groups of participants with and without HPHA dysfunction on gender, age, ethnicity, race, marital status, education or estrogen status (Table 1).

\section{Clinical Features}

HPHA dysfunction was associated with being diagnosed with FM approximately three times longer than those participants with normal HPHA function. Specifically, whereas GH responders had been diagnosed with FM for an average of 3.2 years, the mean time since diagnosis in nonresponders was 9.4 years (Table 2). HPHA dysfunction was also associated with increased pain on the FIQ VAS, increased number of tender-points and higher cumulative myalgic scores, a higher BMI and an increased percentage of body fat $(\mathrm{p}=0.047)$. However, no significant differences were found between the two groups in levels of physical impairment, depression, stiffness, fatigue, sleep quality, overall quality of life or total impact of FM on daily activities. Groups did not differ on serum IGF-1 levels or IGF-1 status (normal versus abnormal for age).

\section{Response to Exhaustive Exercise Testing}

Although all participants were classified as sedentary (engaging in planned exercise for less than 30 minutes a week during the three months prior to the treadmill test), $\mathrm{GH}$ non-responders were significantly less physically fit than GH-responders based on $\mathrm{VO}_{2}$ Max (Table 3). As expected, the workload achieved during the treadmill test was significantly lower in GH non-responders versus responders $(\mathrm{p}=0.001)$. An ANCOVA was preformed to rule out the potential effect of the amount of work performed during the test and percent of body fat content on GH response to exercise. A significant difference in $\mathrm{GH}$ response was maintained between the two groups even after controlling for workload $(p<0.001)$, percentage of body fat $(p=0.001)$, and both simultaneously $(\mathrm{p}=0.006)$.

\section{Biochemical Markers}

Serum IGF-1 levels did not differ between groups (Table 4). Although only marginally significant, pre-exercise cortisol levels tended to be lower in GH non-responders than responders. Non-responders had significantly higher preexercise levels of inflammatory cytokines IL-1 $\alpha$, IL-6, and the chemokine IL- 8 , but not IL-1 $\beta$, IL-1RA, IL-10, or TNF$\alpha$. Since adipose tissue mass can influence the levels of systemic immune response system biomarkers, between group levels were compared while controlling for percentage of body fat. The use of percentage of body fat as a covariate did not influence the observed group differences in IL1- $\alpha$ ( $p$ $=0.034)$, IL-6 $(\mathrm{p}=0.021)$ nor IL-8 $(\mathrm{p}=0.006)$. 
Table 1. Demographic Characteristics of Sample

\begin{tabular}{|c|c|c|c|}
\hline Variable & \multicolumn{3}{|c|}{ Group } \\
\hline Gender (Female) & 7 & 12 & NS \\
\hline Ethnicity & & & NS \\
\hline Marital Status & & & .466 \\
\hline - $\quad$ Married or living together & 6 & 8 & \\
\hline - Divorced or single & 1 & 4 & \\
\hline Education & & & .10 \\
\hline Employed outside the home & & & $.029^{*}$ \\
\hline - $\quad$ Full time & 5 & 3 & \\
\hline - $\quad$ Part Time & 1 & 0 & \\
\hline - $\quad$ Not employed & 1 & 9 & \\
\hline Receiving Disability Payments & & & 1.0 \\
\hline - $\quad$ Yes & 0 & 1 & \\
\hline - $\quad$ No & 7 & 11 & \\
\hline Estrogen Status & & & .147 \\
\hline - $\quad$ Post-menopausal & 1 & 9 & \\
\hline
\end{tabular}
Replacement Therapy. ${ }^{*} \mathrm{p} \leq 0.05$.

\section{Correlations}

Correlations were preformed on demographic characteristics, clinical features and biochemical markers that were found to differ significantly between groups. As no differences existed between groups on fatigue, sleep, depression, stiffness, IL-1 $\beta$, IL-1RA, IL-10 and TNF- $\alpha$, correlational analyses were not performed on these variables. Correlational analyses presented in Table 5 show that 43 correlations were statistically significant at a 0.05 alpha level and were greater than or equal to $r=0.464$. When the Bonferroni-method for correction of multiple comparisons was applied $(0.05 / 14=0.004), 20$ correlations remained significant. As this study was exploratory in nature, the authors leave the interpretation of importance of the 23 correlations that did not remain significant at the adjusted alpha value to the reader.

The number of years diagnosed with FM was positively correlated with BMI $(p=0.041)$ and number of tender points $(p=0.039)$, but was not significant at an adjusted alpha of 0.004 .

Body mass index correlated positively with percent of body fat $(p<0.001)$ and pain as measured by the FIQ VAS $(\mathrm{p}<0.001)$. It inversely correlated with physical endurance as measured by the number of seconds on the treadmill $(\mathrm{p}=$ $0.045)$, physical fitness as measured by $\mathrm{VO}_{2} \operatorname{Max}(\mathrm{p}=$ $0.005)$, serum GH levels immediately following exhaustive exercise $(p=0.004)$, GH levels 1-hour post-exercise $(p=$ $0.003)$ and GH response $(p=0.006)$. Only percentage of body fat, pain, and $\mathrm{GH}$ levels at 1-hour post-exercise remained significant at the adjusted alpha level of 0.004 .

Percentage of body fat was inversely related to physical endurance $(\mathrm{p}=0.038)$, physical fitness $(\mathrm{p}=0.002)$, GH postexercise $(p=0.023)$, GH 1-hour post-exercise $(p=0.008)$, and $\mathrm{GH}$ response $(\mathrm{p}=0.027)$. Only physical fitness and $\mathrm{GH}$ levels at 1-hour post-exercise remained significant at the adjusted alpha level of .004 . 
Table 2. Clinical Features of Sample

\begin{tabular}{|c|c|c|c|}
\hline Variable & \multicolumn{2}{|c|}{ Group } & $\begin{array}{l}\text { Independent } t-T e s t \\
\text { (2-Tailed) }\end{array}$ \\
\hline Years diagnosed with FM & $3.2 \pm 3.0$ & $9.4 \pm 5.7$ & $0.008^{* *}$ \\
\hline BDI-R Score & $9.1 \pm 6.3$ & $8.3 \pm 7.9$ & 0.816 \\
\hline FIQ Pain VAS Score & $4.1 \pm 2.1$ & $6.5 \pm 1.9$ & $0.024^{*}$ \\
\hline FIQ Fatigue VAS Score & $6.6 \pm 2.7$ & $7.4 \pm 1.6$ & 0.395 \\
\hline FIQ Sleep VAS Score & $6.7 \pm 3.9$ & $8.1 \pm 1.5$ & 0.398 \\
\hline FIQ Stiffness VAS Score & $5.9 \pm 2.7$ & $7.7 \pm 2.1$ & 0.123 \\
\hline Number of Tender Points & $15.9 \pm 1.8$ & $17.6 \pm 1.0$ & $0.014^{*}$ \\
\hline Cumulative Myalgic Score & $28.4 \pm 6.8$ & $40.5 \pm 5.8$ & $0.001^{* * *}$ \\
\hline Body Mass Index & $27.3 \pm 6.1$ & $33.3 \pm 4.5$ & $0.025^{*}$ \\
\hline Percent Fat Mass & $24.5 \pm 9.7$ & $34.4 \pm 9.4$ & $0.047^{*}$ \\
\hline Percent Lean Muscle Mass & $51.7 \pm 5.8$ & $57.3 \pm 5.5$ & 0.054 \\
\hline IGF-1 Status (normal vs abnormal for age) & $3 v s 4$ & $6 v s 6$ & 1.0 \\
\hline
\end{tabular}

Responders $=\mathrm{GH}$ response to exercise of $\geq 5 \mathrm{ng} / \mathrm{mL}$. Non-responders $=\mathrm{GH}$ response to exercise of $0 \mathrm{ng} / \mathrm{mL}$. SD $=$ Standard Deviation. BDI-R $=$ Beck Depression InventoryRevised. FIQ = Fibromyalgia Impact Questionnaire. VAS $=$ Visual Analogue Scale. IGF-1 $=$ Insulin-like Growth Factor-1. $* \mathrm{p} \leq 0.05 . * * \mathrm{p} \leq 0.01 . * * * \mathrm{p} \leq 0.001$.

Table 3. Growth Hormone Response to Exhaustive Exercise

\begin{tabular}{|l|c|c|c|}
\hline \multirow{2}{*}{ Variable } & \multicolumn{2}{|c|}{ Group } & \multirow{2}{*}{ Independent t-Test (2-Tailed) } \\
\cline { 2 - 4 } & Responders (n=7) \pm SD & Non-Responders (n= 12) \pm SD & \\
\hline \hline Growth Hormone (ng/mL) & & & 0.251 \\
- Pre-exercise & $0.6 \pm 0.5$ & $1.9 \pm 3.6$ & $0.007^{*}$ \\
- Post-exercise $\quad 1$-hr. Post-exercise & $12.2 \pm 7.6$ & $0.7 \pm 1.5$ & $0.048^{*}$ \\
- Response & $1.0 \pm 0.8$ & $0.2 \pm 0.2$ & $0.006^{*}$ \\
\hline Physical Fitness-VO ${ }_{2}$ Max (Units) & $11.6 \pm 7.3$ & 0 & $0.001^{* * *}$ \\
\hline Physical Endurance (Seconds on TM) & $24.6 \pm 3.9$ & $18.2 \pm 3.0$ & $<0.001^{* * *}$ \\
\hline Workload (kJ) & $988.3 \pm 200.4$ & $446.3 \pm 226.5$ & $0.001^{* * *}$ \\
\hline
\end{tabular}

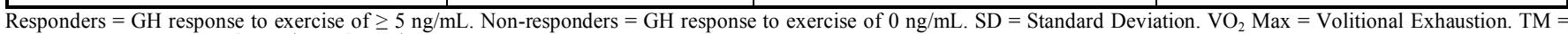
Treadmill. Response $=\mathrm{GH}^{\text {Post exercise }}-\mathrm{GH}^{\text {Pre-exercise }} . * \mathrm{p} \leq 0.05 . * * \mathrm{p} \leq 0.001$.

Pain, as measured by the FIQ VAS, correlated strongly with BMI $(\mathrm{p}<0.001)$, but not percentage of body fat $(\mathrm{p}=$ $0.271)$. The more pain one had, the less physical endurance they had $(p=0.002)$ and the less physically fit they were ( $p$ $=0.010$ ). Pain as measured by the number of tender points strongly correlated with CMS scores $(p>0.001)$ and moderately with number of years diagnosed with FM ( $\mathrm{p}=$ $0.039)$. The number of tender points inversely correlated with physical endurance $(\mathrm{p}=0.034)$, physical fitness $(\mathrm{p}=$ $0.008)$, GH levels immediately post-exercise $(p=0.011)$, and GH response $(\mathrm{p}=0.019)$.
Pain as measured by CMS scores positively correlated with number of years diagnosed with FM $(p=0.032)$ and number of tender points $(\mathrm{p}<0.001)$. CMS scores inversely correlated with physical endurance $(\mathrm{p}=0.008)$, physical fitness $(\mathrm{p}=0.003), \mathrm{GH}$ levels immediately post-exercise $(\mathrm{p}=$ $0.001)$ and $\mathrm{GH}$ response $(\mathrm{p}=0.002)$.

Physical endurance inversely correlated with all three pain measures: FIQ VAS scores $(\mathrm{p}=0.002)$; CMS scores ( $\mathrm{p}$ $=0.008)$; and number of tender points $(\mathrm{p}=0.034)$. Physical endurance was also inversely correlated with IL-1A ( $p=$ $0.043)$, IL-6 ( $p=0.045)$, IL-8 ( $=0.032)$, serum GH levels immediately following exhaustive exercise $(p=0.003)$ and 
Table 4. Biochemical Marker Differences between Groups

\begin{tabular}{|c|c|c|c|}
\hline Cortisol (pg/mL) & $0.513 \pm 0.181$ & $0.346 \pm 0.198$ & 0.085 \\
\hline $\mathrm{IL}-1 \alpha(\mathrm{pg} / \mathrm{mL})$ & $234.3 \pm 170.2$ & $597.7 \pm 435.5$ & $0.021^{* *}$ \\
\hline IL-6 & $96.2 \pm 83.8$ & $257.7 \pm 164.9$ & $0.012^{*}$ \\
\hline IL-8 & $17.0 \pm 14.5$ & $51.7 \pm 30.3$ & $0.004^{* *}$ \\
\hline IL-10 & $1.0 \pm 2.7$ & $12.9 \pm 30.4$ & 0.323 \\
\hline TNF- $\alpha$ & $5.8 \pm 7.5$ & $9.2 \pm 13.3$ & 0.540 \\
\hline
\end{tabular}

Responders $=\mathrm{GH}$ response to exercise of $\geq 5 \mathrm{ng} / \mathrm{mL}$. Non-responders $=$ GH response to exercise of $0 \mathrm{ng} / \mathrm{mL}$. SD $=$ Standard Deviation. ${ }^{*} \mathrm{p} \leq 0.05 .{ }^{* *} \mathrm{p} \leq 0.01$.

at 1 -hour post-exercise $(\mathrm{p}=0.001)$, and $\mathrm{GH}$ response $(\mathrm{p}=$ $0.005)$.

Physical fitness positively correlated with physical endurance $(\mathrm{p}<0.001)$, GH levels immediately post-exercise $(\mathrm{p}<0.001)$ and at 1 -hour post-exercise $(\mathrm{p}<0.001)$ and GH response $(\mathrm{p}<0.001)$. It inversely correlated with BMI $(\mathrm{p}=$ $0.005)$, percentage of body fat $(\mathrm{p}=0.002)$ and all three pain measures: FIQ VAS scores $(\mathrm{p}=0.010)$; CMS scores $(\mathrm{p}=$ $0.003)$; and number of tender points $(\mathrm{p}=0.008)$.

Serum levels of GH immediately following exercise positively correlated physical endurance $(p=0.003)$, physical fitness $(p=0.001)$, GH levels one hour postexercise $(p=0.007)$ and $G H$ response $(p<0.001)$. It inversely correlated with BMI $(p=0.004)$ and percentage of body fat $(p=0.023)$, number of tender points $(p=0.011)$ and CMS scores $(\mathrm{p}=0.001)$.
Serum levels of GH 1-hour post-exercise positively correlated with physical endurance $(p=0.001)$, physical fitness $(\mathrm{p}<0.001), \mathrm{GH}$ levels immediately post-exercise $(\mathrm{p}=$ $0.007)$ and $\mathrm{GH}$ response $(\mathrm{p}=0.006)$.

Growth hormone response positively correlated with physical endurance $(p=0.005)$, physical fitness $(p<0.001)$, GH levels immediately post-exercise $(p<0.001)$ and GH 1 hour post-exercise $(p=0.006)$. It inversely correlated with BMI $(p=0.006)$, percentage of body fat $(p=0.027)$, number of tender points $(p=0.019)$ and CMS scores $(p=0.002)$.

All of the inflammatory biomarkers directly correlated with each other: IL- $1 \alpha$ with IL-6 $(\mathrm{p}=0.003)$ and IL-8 $(\mathrm{p}=$ $0.002)$. Physical endurance negatively correlated with IL-1 $\alpha$ $(p=0.043)$, IL-6 $(p=0.045)$, and IL-8 $(p=0.032)$. IL-8 negatively correlated with $\mathrm{GH}$ response $(\mathrm{p}=0.027)$. In effect, there were no other significant correlations between

Table 5. Correlations of Statistically Significant Demographic Characteristics, Clinical Features and Biochemical Markers

\begin{tabular}{|c|c|c|c|c|c|c|c|c|c|c|c|c|c|c|}
\hline 1. Years Diagnosed with FM & - & & & & & & & & & & & & & \\
\hline 3. \% Body Fat & $.488^{*}$ & $.898 * * *$ & - & & & & & & & & & & & \\
\hline 4. Pain - FIQ VAS & .225 & $.952 * * *$ & .266 & - & & & & & & & & & & \\
\hline 6. Pain - CMS & $.506^{*}$ & .429 & .361 & .389 & $.858 * * *$ & - & & & & & & & & \\
\hline 7. Physical Endurance & -.268 & $-.468 *$ & $-.479 *$ & $-.662 * *$ & $-.488^{*}$ & $-.592 * *$ & - & & & & & & & \\
\hline 8. $\mathrm{VO}_{2} \mathrm{Max}$ & -.305 & $-.621 * *$ & $-.668^{* *}$ & $-.577 * *$ & $-.589 * *$ & $-.637 * *$ & $.813^{* * * *}$ & - & & & & & & \\
\hline 9. GH Post-exercise & -.316 & $-.631 * *$ & $-.519^{*}$ & -.412 & $-.570^{*}$ & $.697 * *$ & $.647 * *$ & $.747 * * *$ & - & & & & & \\
\hline 13. IL-6 & -.169 & -.027 & -.009 & .403 & .094 & .186 & $-.464^{*}$ & -.349 & -.338 & -.164 & -.392 & $.639 * *$ & - & \\
\hline 14. IL-8 & .028 & -.014 & .022 & .367 & .196 & .330 & $-.492 *$ & -.442 & -.454 & -.184 & $-.506^{*}$ & $.669^{* * *}$ & $.897 * * *$ & - \\
\hline
\end{tabular}

FIQ $=$ Fibromyalgia Impact Questionnaire. TP $=$ Tender points. CMS $=$ Cumulative Myalgic Score. TM $=$ Treadmill. $* \mathrm{p} \leq 0.05 .{ }^{* *} \mathrm{p} \leq 0.01 .{ }^{* * *} \mathrm{p} \leq 0.001$. 
inflammatory biomarkers and demographic characteristics or other clinical features of FM in this sample.

\section{DISCUSSION}

The primary aim of our study was to determine if serum cytokine levels and FM symptom severity would be higher in FM patients with defective GH response to exhaustive exercise compared to those with normal HPHA function. Our data support the idea that, within the context of FM, a defective GH response to the stress of acute exercise is associated with elevated resting levels of some inflammatory cytokines and levels of pain, irrespective of BMI, adiposity, workload, or physical fitness level.

A secondary aim of this study was to explore the relationship between elevated immune response system biomarkers and symptom severity in persons diagnosed with FM with and without concurrent HPHA dysfunction [52, 53]. Five studies in FM have suggested that inflammatory cytokines and chemokines may be associated with FM symptomology [31-35]. Wallace found elevated serum levels of IL-8 in FM and noted that IL-8 increased with the duration of FM symptoms [34]. Subsequent studies reported similar increases in IL-8 in the sera of FM patients and a direct relationship between IL-8 levels and pain severity [5456]. Analogous to this previous research, we found elevated serum levels of IL-8 in addition to elevated IL- $1 \alpha$ and IL-6. While increased levels of TNF- $\alpha$, and IL-10 have been observed in FM [31, 32, 34, 35, 37], this observation has not been consistent throughout all studies [36, 37] and was not supported by our data.

Based on prior observations, researchers have postulated there may be a temporal relationship between increased proinflammatory cytokines and FM symptoms [34]. Similarly, our data suggested that cytokine dysregulation is associated with increased pain severity as well as GH dysfunction. Specifically, participants with HPHA dysfunction who reported higher levels of pain had been diagnosed with FM approximately 6 years longer than those with normal GH response to exhaustive exercise. This observation supports the notion that neuroendocrine dysfunction in FM is not a static state, but rather is progressive in nature; a finding reminiscent of Selye's research on adrenal response to chronic stress [57]. Consistent with this idea is our finding that GH non-responders tended to have lower serum cortisol levels than responders.

Correlational analyses revealed significant associations between many variables of interest. Most significantly, participants with poor GH response had higher pain levels, lower physical endurance and poorer fitness levels. In addition, increased pain severity was associated with increased weight (BMI) and greater physical dysfunction (physical endurance and fitness levels). Contrary to the a priori assumptions, our data did not show any correlation between inflammatory markers and pain. It has been suggested in genetic studies that the use of the Bonferroni method of corrections for multiple comparisons may be too stringent of a correction for alpha levels in biochemical correlations, thus significant associations may be missed [58]. This issue needs to be more thoroughly evaluated in FM research and possibly incorporated in future studies. Despite the lack of a statistically significant correlation between pain and inflammatory markers, the previously discussed t-tests provide some preliminary evidence that may link pain and immune system response with HPHA dysfunction in FM.

This exploratory analysis study has a number or limitations that should be considered when interpreting the findings. First, $90 \%$ of the sample from the parent study did not mount a normal GH response to exhaustive exercise thus limited the sample size to 12 participants with normal GH function and a comparison group of 12 participants with abnormal GH response. In addition, after exclusion of the five samples that were not of sufficient quality or quantity to be analyzed, the statistical power to detect correlations between groups on biochemical markers and FM symptoms was significantly decreased thus increasing the risk of not detecting meaningful differences between groups. Likewise, the small sample size may have also limited our ability to discern significant associations among biomarkers and FM symptoms. Therefore, it is unclear if these findings would generalize to all persons with FM who exhibit a normal GH response to exercise. Furthermore, our sample was comprised of a homogenous group of women, thus results may not be representative of men and minorities. Although it is not known if the prevalence of abnormal GH response to exercise in FM patients managed in primary care differs from those seen in a tertiary care setting, it is possible and thus needs to be evaluated in future research. While groups did not differ on estrogen status, we are unable to rule out the potential confounding effects of exact estradiol levels on $\mathrm{GH}$ response. As the categorization of menopausal status was based on self-report of time from last menses, date of surgically induced menopause, or presence of hormone replacement therapy versus estradiol levels, this measure did not address the other features of menopause such as fatigue, mood, and sleep separate from FM. Therefore it is not known if GH data from this study are related to estradiol levels, a measure that will be critical in follow up studies. Another limitation in this trial is that it did not include healthy controls which could have provided comparison levels for the cytokine profiles.

Our data support the idea that within the context of FM a defective GH response to the stress of acute exercise is associated with elevated resting levels of some inflammatory cytokines and pain level. One potential confounder in our results is the fact that the $\mathrm{GH}$ responders were more physically fit than non-responders, as evidenced by the differences in $\mathrm{VO}_{2} \mathrm{Max}$ and total work performed during the treadmill test. Thus it is possible that difference in fitness level may have influenced resting cytokine levels, independently of GH status, as was reported in one previous study [53]. Mitigating this explanation for our results is the observation that the resting levels of IL-1 $\alpha$, IL- 6 and IL-8 remained significantly elevated in $\mathrm{GH}$ non-responders even after controlling for workload or $\mathrm{VO}_{2} \mathrm{Max}$. It is evident that future studies should specifically examine the relationship between inflammatory markers in FM and healthy controls matching for fitness level and the GH response to exercise. Furthermore, un-stimulated cortisol levels may have been insensitive to distinguishing HPA differences between the 2 groups. Future studies may be designed to include the use of the combined dexamethasone suppression/corticotropinreleasing hormone stimulation test, as it is significantly more 
sensitive and specific than the unchallenged measurement of cortisol.

\section{CONCLUSIONS}

The results reported herein suggest that a defective growth hormone response to exercise may be associated with increased levels of blood cytokines and pain severity in FM. Although this study produced some interesting preliminary findings, cautious interpretation is warranted until these findings can be replicated in a larger, more heterogeneous sample.

\section{ACKNOWLEDGEMENTS}

R.L.R. is funded by a Post-doctoral Fellowship Award from the National Institutes of Health and is a SubInvestigator on pharmaceutical clinical trials with Schwarz Biosciences, Jazz Pharmaceuticals, and Pfizer, Incorporated.

K.D.J. is funded by grants from the National Institutes of Health, the UniHealth Foundation, the Fibromyalgia Information Foundation, and is a Co-principal Investigator on pharmaceutical clinical trials with Schwarz Biosciences, Jazz Pharmaceuticals, and Pfizer, Incorporated.

R.M.B. is funded by grants from the National Institutes of Health, the Fibromyalgia Information Foundation, and is a Principal Investigator on pharmaceutical clinical trials with Schwarz Biosciences, Jazz Pharmaceuticals, and Pfizer, Incorporated.

R.L.W. is currently funded by grants from Schwarz Biosciences, Jazz Pharmaceuticals, and Pfizer, Incorporated.

B.J.D. is funded by grants from the National Cancer Institute, a Specialized Center of Research Award from the Leukemia and Lymphoma Society, a Clinical Scientist Award from the Burroughs Wellcome Fund, and the T. J. Martell Foundation.

L.J.W. is funded by grants from the American Cancer Society and the National Institutes of Health.

The authors would like to acknowledge Anthony Potter and Christine Nelson for their diligent attention to manuscript preparation.

\section{REFERENCES}

[1] Clauw DJ, Crofford LJ. Chronic widespread pain and fibromyalgia: what we know, and what we need to know. Best Pract Res Clin Rheumatol 2003; 17: 685-701.

[2] Price DD, Staud R. Neurobiology of fibromyalgia syndrome. J Rheumatol Suppl 2005; 75: 22-8.

[3] Yunus MB. Central sensitivity syndromes: a new paradigm and group nosology for fibromyalgia and overlapping conditions, and the related issue of disease versus illness. Semin Arthritis Rheum 2008; 37: 339-52.

[4] Adler GK, Geenen R. Hypothalamic-pituitary-adrenal and autonomic nervous system functioning in fibromyalgia. Rheum Dis Clin North Am 2005; 31: 187-202, xi.

[5] Martinez-Lavin M, Hermosillo AG. Autonomic nervous system dysfunction may explain the multisystem features of fibromyalgia [editorial; comment]. Semin Arthritis Rheum 2000; 29: 197-9.

[6] Dadabhoy D, Crofford LJ, Spaeth M, Russell IJ, Clauw DJ. Biology and therapy of fibromyalgia. Evidence-based biomarkers for fibromyalgia syndrome. Arthritis Res Ther 2008; 10: 211.

[7] Crofford LJ, Young EA, Engleberg NC, et al. Basal circadian and pulsatile ACTH and cortisol secretion in patients with fibromyalgia and/or chronic fatigue syndrome. Brain Behav Immun 2004; 18: 314-25.
[8] McBeth J, Chiu YH, Silman AJ, et al. Hypothalamic-pituitaryadrenal stress axis function and the relationship with chronic widespread pain and its antecedents. Arthritis Res Ther 2005; 7: R992-R1000.

[9] Jones KD, Deodhar P, Lorentzen A, Bennett RM, Deodhar AA. Growth hormone perturbations in fibromyalgia: a review. Semin Arthritis Rheum 2007; 36: 357-79.

[10] Bennett RM, Cook DM, Clark SR, Burckhardt CS, Campbell SM. Hypothalamic-pituitary-insulin-like growth factor-I axis dysfunction in patients with fibromyalgia. J Rheumatol 1997; 24: 1384-9.

[11] Bennett RM. Adult growth hormone deficiency in patients with fibromyalgia. Curr Rheumatol Rep 2002; 4: 306-12.

[12] Lorton D, Lubahn CL, Zautra AJ, Bellinger DL. Proinflammatory cytokines and sickness behavior in rheumatic diseases. Curr Pharm Des 2008; 14: 1242-60.

[13] Wigers SH. [Fibromyalgia--an update]. Tidsskr Nor Laegeforen 2002; 122: 1300-4.

[14] Dantzer R. Cytokine-induced sickness behaviour: a neuroimmune response to activation of innate immunity. Eur J Pharmacol 2004; 500: 399-411.

[15] Connor TJ, Leonard BE. Depression, stress and immunological activation: the role of cytokines in depressive disorders. Life Sci 1998; 62: 583-606.

[16] Kiecolt-Glaser JK, Preacher KJ, MacCallum RC, Atkinson C, Malarkey WB, Glaser R. Chronic stress and age-related increases in the proinflammatory cytokine IL-6. Proc Natl Acad Sci USA 2003; 100: 9090-5.

[17] Ostrowski K, Rohde T, Asp S, Schjerling P, Pedersen BK. Pro- and anti-inflammatory cytokine balance in strenuous exercise in humans. J Physiol 1999; 515: 287-91.

[18] Smith LL. Cytokine hypothesis of overtraining: a physiological adaptation to excessive stress? Med Sci Sports Exerc 2000; 32: 317-31.

[19] Vgontzas AN, Papanicolaou DA, Bixler EO, Kales A, Tyson K, Chrousos GP. Elevation of plasma cytokines in disorders of excessive daytime sleepiness: role of sleep disturbance and obesity [see comments]. J Clin Endocrinol Metab 1997; 82: 1313-6.

[20] Konsman JP, Parnet P, Dantzer R. Cytokine-induced sickness behaviour: mechanisms and implications. Trends Neurosci 2002; 25: 154-9.

[21] Park HS, Park JY, Yu R. Relationship of obesity and visceral adiposity with serum concentrations of CRP, TNF-alpha and IL-6. Diabetes Res Clin Pract 2005; 69: 29-35.

[22] Suzuki K, Nakaji S, Yamada M, Totsuka M, Sato K, Sugawara K. Systemic inflammatory response to exhaustive exercise. Cytokine kinetics. Exerc Immunol Rev 2002; 8: 6-48.

[23] Suzuki K, Nakaji S, Yamada M, et al. Impact of a competitive marathon race on systemic cytokine and neutrophil responses. Med Sci Sports Exerc 2003; 35: 348-55.

[24] Wong CM. Post-traumatic stress disorder: advances in psychoneuroimmunology. Psychiatr Clin North Am 2002; 25: 36983 , vii.

[25] Sjoholm A, Nystrom T. Inflammation and the etiology of type 2 diabetes. Diabetes Metab Res Rev 2006; 22: 4-10.

[26] Hayley S, Poulter MO, Merali Z, Anisman H. The pathogenesis of clinical depression: stressor- and cytokine-induced alterations of neuroplasticity. Neuroscience 2005; 135: 659-78.

[27] Angst MS, Clark JD, Carvalho B, Tingle M, Schmelz M, Yeomans DC. Cytokine profile in human skin in response to experimental inflammation, noxious stimulation, and administration of a COXinhibitor: a microdialysis study. Pain 2008; 139: 15-27.

[28] Kleibeuker W, Gabay E, Kavelaars A, et al. IL-1 beta signaling is required for mechanical allodynia induced by nerve injury and for the ensuing reduction in spinal cord neuronal GRK2. Brain Behav Immun 2008; 22: 200-8.

[29] Summer GJ, Romero-Sandoval EA, Bogen O, Dina OA, Khasar SG, Levine JD. Proinflammatory cytokines mediating burn-injury pain. Pain 2008; 135: 98-107.

[30] Morrow JD, Opp MR. Diurnal variation of lipopolysaccharideinduced alterations in sleep and body temperature of interleukin-6deficient mice. Brain Behav Immun 2005; 19: 40-51.

[31] Bazzichi L, Rossi A, Massimetti G, et al. Cytokine patterns in fibromyalgia and their correlation with clinical manifestations. Clin Exp Rheumatol 2007; 25: 225-30. 
[32] Gur A, Karakoc M, Nas K, et al. Cytokines and depression in cases with fibromyalgia. J Rheumatol 2002; 29: 358-61.

[33] Maes M, Libbrecht I, Van Hunsel F, et al. The immuneinflammatory pathophysiology of fibromyalgia: increased serum soluble gp130, the common signal transducer protein of various neurotrophic cytokines [In Process Citation]. Psychoneuroendocrinology 1999; 24: 371-83.

[34] Wallace DJ, Linker-Israeli M, Hallegua D, Silverman S, Silver D, Weisman MH. Cytokines play an aetiopathogenetic role in fibromyalgia: a hypothesis and pilot study. Rheumatology (Oxford) 2001; 40: 743-9.

[35] Wang H, Moser M, Schiltenwolf M, Buchner M. Circulating cytokine levels compared to pain in patients with fibromyalgia -- a prospective longitudinal study over 6 months. J Rheumatol 2008; 35: 1366-70.

[36] Amel MRKD, Swinden D, Todd I, Powell RJ. Normal production of inflammatory cytokines in chronic fatigue and fibromyalgia syndromes determined by intracellular cytokine staining in shortterm cultured blood mononuclear cells. Clin Exp Immunol 2003; 132: $360-5$.

[37] Uceyler N, Valenza R, Stock M, Schedel R, Sprotte G, Sommer C. Reduced levels of antiinflammatory cytokines in patients with chronic widespread pain. Arthritis Rheum 2006; 54: 2656-64.

[38] Jones KD, Deodhar AA, Burckhardt CS, Perrin NA, Hanson GC, Bennett RM. A combination of 6 months of treatment with pyridostigmine and triweekly exercise fails to improve insulin-like growth factor-I levels in fibromyalgia, despite improvement in the acute growth hormone response to exercise. J Rheumatol 2007; 34 : 1103-11.

[39] Paiva ES, Deodhar A, Jones KD, Bennett R. Impaired growth hormone secretion in fibromyalgia patients: evidence for augmented hypothalamic somatostatin tone. Arthritis Rheum 2002; 46: 1344-50.

[40] Elenkov IJ, Chrousos GP. Stress hormones, proinflammatory and antiinflammatory cytokines, and autoimmunity. Ann N Y Acad Sci 2002; 966: 290-303.

[41] Greenhalgh CJ, Alexander WS. Suppressors of cytokine signalling and regulation of growth hormone action. Growth Horm IGF Res 2004; 14: 200-6.

[42] Redelman D, Welniak LA, Taub D, Murphy WJ. Neuroendocrine hormones such as growth hormone and prolactin are integral members of the immunological cytokine network. Cell Immunol 2008; 252: 111-21.

[43] Wolfe F, Smythe HA, Yunus MB, et al. The American College of Rheumatology 1990 Criteria for the Classification of Fibromyalgia.
Report of the Multicenter Criteria Committee. Arthritis Rheum 1990; 33: 160-72.

[44] Burckhardt CS, Clark SR, Padrick KP. Use of the modified Balke treadmill protocol for determining the aerobic capacity of women with fibromyalgia. Arthritis Care Res 1989; 2: 165-7.

[45] Beck A, Steer R. Manual for the Beck Depression Inventory. San Antonio, Texas: Psychological Corporation 1987.

[46] Burckhardt CS, O'Reilly CA, Wiens AN, Clark SR, Campbell SM, Bennett RM. Assessing depression in fibromyalgia patients. Arthritis Care Res 1994; 7: 35-9.

[47] Bennett RM, Schein J, Kosinski MR, Hewitt DJ, Jordan DM, Rosenthal NR. Impact of fibromyalgia pain on health-related quality of life before and after treatment with tramadol/acetaminophen. Arthritis Rheum 2005; 53: 519-27.

[48] Flanagan JC. Measurement of quality of life: current state of the art. Arch Phys Med Rehabil 1982; 63: 56-9.

[49] Burckhardt CS, Woods SL, Schultz AA, Ziebarth DM. Quality of life of adults with chronic illness: a psychometric study. Res Nurs Health 1989; 12: 347-54.

[50] Burckhardt CS, Clark SR, Bennett RM. The fibromyalgia impact questionnaire: development and validation. J Rheumatol 1991; 18 : 728-33.

[51] Wilmore JH. Body breadth equipment and measurement techniques. Champaign, IL: Human Kinetics 1988.

[52] Molitch ME, Clemmons DR, Malozowski S, et al. Evaluation and treatment of adult growth hormone deficiency: an Endocrine Society Clinical Practice Guideline. J Clin Endocrinol Metab 2006; 91: 1621-34.

[53] Thomas NE, Williams DR. Inflammatory factors, physical activity, and physical fitness in young people. Scand J Med Sci Sports 2008; 18: 543-56.

[54] Bennett RM, Clark SR, Goldberg L, et al. Aerobic fitness in patients with fibrositis. A controlled study of respiratory gas exchange and 133xenon clearance from exercising muscle. Arthritis Rheum 1989; 32: 454-60.

[55] Armstrong RB, Ogilvie RW, Schwane JA. Eccentric exerciseinduced injury to rat skeletal muscle. J Appl Physiol 1983; 54: 8093.

[56] Lieber RL, Friden J. Morphologic and mechanical basis of delayedonset muscle soreness. J Am Acad Orthop Surg 2002; 10: 67-73.

[57] Selye H. A syndrome produced by diverse nocuous agents. 1936. J Neuropsychiatry Clin Neurosci 1998; 10: 230-1.

[58] Narum SR. Beyond Bonferroni: less conservative analyses for conservation genetics. Conserv Genet 2006; 7: 783-7.

This is an open access article licensed under the terms of the Creative Commons Attribution Non-Commercial License (http://creativecommons.org/licenses/by$\mathrm{nc} / 3.0 /$ ) which permits unrestricted, non-commercial use, distribution and reproduction in any medium, provided the work is properly cited. 